\title{
Découverte en Corse de Matsucoccus feytaudi Duc (Homoptera: Margarodidae), cochenille du pin maritime
}

\author{
H Jactel, P Ménassieu, C Burban \\ Laboratoire d'entomologie forestiere, station de recherches forestières, \\ domaine de l'Hermitage, Inra, BP 45, 33611 Gazinet cedex, France
}

(Reçu le 10 octobre 1994 ; accepté le 13 mars 1995)

\begin{abstract}
Résumé - Une enquête portant sur la présence de la cochenille du pin maritime Matsucoccus feytaudi Duc en Corse a été réalisée en 1994, à l'aide d'un piègeage sexuel. Au total, 24 peuplements forestiers ont été suivis, répartis dans tous les massifs de pin maritime de l'île. Une population de cochenilles a été découverte dans la forêt de Pineto (Haute-Corse), alors que ce ravageur était jusque là déclaré absent de l'île. L'origine de cette population, indigène ou d'introduction récente, est discutée et les risques de pullulation évalués.
\end{abstract}

Homoptera / Margarodidae / Matsucoccus feytaudi / Pinus pinaster / piégeage sexuel / aire de répartition / risques de pullulation

Summary - Detection of the maritime pine bast scale, Matsucoccus feytaudi Duc (Homoptera: Margarodidae), in Corsica. In 1994, a survey of the maritime pine bast scale, Matsucoccus feytaudi Duc, in Corsica was carried out by pheromone trapping. Twenty-four stands were investigated, located in all the maritime pine forests of the island. One population of maritime pine scale was discovered in the Forêt de Pineto (Upper Corsica), although this pest was declared absent from the island until then. The origin of the population, indigenous or recently introduced, was discussed and the risks of outbreak estimated.

Homoptera / Margarodidae / Matsucoccus feytaudi / Pinus pinaster / sexual trapping / distribution area / risk of outbreak 


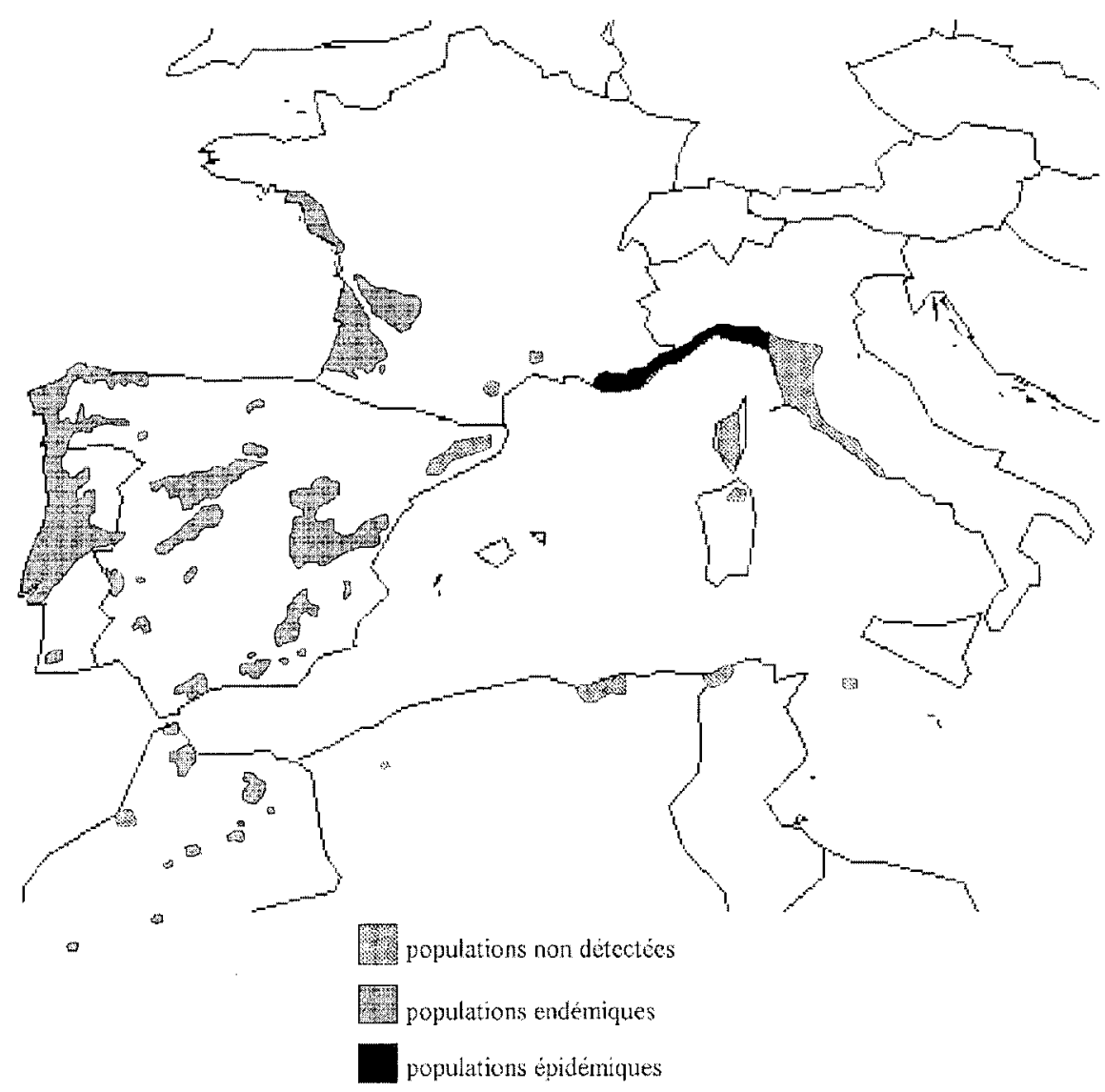

Fig 1. État des connaissances en 1988 sur la distribution géographique de Matsucoccus feytaudi Duc dans l'aire naturelle du pin maritime (d'après Destremau, 1974 ; Riom et Gerbinot 1977 et Baronio et Butturini, 1988).

\section{INTRODUCTION}

La cochenille Matsucoccus feytaudi Duc est un ravageur endémique du pin maritime, Pinus pinaster Ait, dans toute la partie occidentale de l'aire naturelle de cette essence forestière (fig 1), et n'y commet aucun dégât notable (Riom et Gerbinot, 1977). En revanche cet insecte, présent à l'état épidémique dans le sud-est de la France depuis la fin des années 1950, est à l'ori- gine de la destruction de plus de 120000 ha de pins maritimes, principalement dans les massifs des Maures et de l'Esterel (Schvester, 1971). Introduit dans le nord de l'Italie à la fin des années 1970, il présente actuellement des pullulations en Ligurie et dans la partie septentrionale de la Toscane (Baronio et Butturini, 1988). L'extension des infestations vers la limite orientale de l'aire du pin maritime semble donc se poursuivre, avec une vitesse de progression de 5 à $10 \mathrm{~km}$ par an, c'est-à-dire conforme à celle enre- 
gistrée au début de l'épidémie (Schvester, 1971).

La question de l'introduction de $M$ feytaudi en Corse préoccupe depuis plusieurs années les responsables forestiers. Cette région, proche des zones où sévit l'épidémie, abrite en effet plus de 27000 ha de pins maritimes. Caractérisés par des critères morphologiques remarquables, branches fines et rectitude du tronc, ces arbres constituent des génotypes particulièrement recherchés. Ils sont d'ores et déjà inclus dans un schéma d'amélioration génétique du pin maritime, fondé sur l'hybridation des races corse et landaise (Baradat et Pastuszka, 1992). Leur destruction serait donc dommageable non seulement pour la forêt corse mais également pour le patrimoine génétique de l'espèce. Or le risque de développement d'une épidémie faisant suite à l'introduction de la cochenille dans l'île s'avère d'autant plus important que la race corse de pin maritime est signalée, en tests comparatifs implantés en zone épidémique (Provence), comme une des plus sensibles à l'insecte (Schvester et Ughetto, 1986 ; Harfouche et al, 1994).

Jusqu'en 1992, des prospections ont été effectuées en Corse pour détecter la présence de $M$ feytaudi. Basées sur la capture de cochenilles femelles dans des bandes pièges en carton ondulé appliquées sur le tronc (Carle, 1968), elles se sont toujours avérées négatives, au point que la Corse a obtenu le statut de zone protégée vis-à-vis de cet insecte nuisible (arrêté du 2 septembre 1993 de l'Union européenne). Une incertitude pesait néanmoins sur ces résultats, liée à la difficulté de mise en œuvre de la méthode (Riom, 1979) et à son efficacité limitée dans le cas d'arbres faiblement infestés. L'identification de la phéromone sexuelle de $M$ feytaudi (Einhorn et al, 1990) puis la démonstration de son efficacité en zone endémique (Jactel et al, 1994) ont montré depuis que le piégeage sexuel constituait une méthode plus puissante pour détecter la présence de la cochenille dans des zones à faibles densités de population.

Une enquête par piégeage sexuel a donc été entreprise en Corse, en 1994, pour repérer la présence éventuelle de $M$ feytaudi, décrire sa répartition sur l'île et donner des premières estimations de ses niveaux de population.

\section{MATÉRIEL ET MÉTHODES}

$M$ feytaudi est une cochenille inféodée à une seule espèce de végétal hôte, $P$ pinaster (Rieux, 1975) Le choix des sites de piégeage s'est donc d'abord établi en fonction de l'aire de répartition de cette essence forestière en Corse. Le pin maritime présente une répartition très morcelée dans cette région, les deux tiers de sa surface se situant en Corse-du-Sud (fig 2). II a donc été décidé, en concertation avec les services impliqués dans l'enquête (Département de la santé des forêts, Office national des forêts, Service de la protection des végétaux, Service régional de la forêt et du bois de la Direction régionale de l'agriculture et de la forêt, Direction départementale de l'agriculture et de la forêt de Haute-Corse et Corse-du-Sud), d'installer au moins un piège dans chacun des massifs identifiés, soit en futaie, soit en zone de maquis lorsque le pin maritime y était l'essence prépondérante. Afin de repérer d'éventuelles introductions récentes, notamment par transport de matériel végétal infesté, un certain nombre de pièges ont également été mis en place près des zones portuaires, c'est-à-dire dans des peuplements proches de Bastia, Calvi, Porto-Vecchio, Solenzara et Bonifacio, ainsi que sur les principaux axes routiers (fig 2). Au total, 24 pièges à phéromones ont donc été installés, dans des peuplements de pin maritime dont le type forestier, l'altitude, et la situation géographique sont précisés au tableau I.

Pour piéger les mâles ailés de $M$ feytaudi, des pièges à glu ont été utilisés. Ils sont constitués d'une plaque de polypropylène blanc de $400 \mathrm{~cm}^{2}$, recouverte de plusieurs feuilles plastique transparentes et engluées. À chaque relevé, la feuille transparente supérieure, souvent saturée d'insectes collés, est enlevée, libérant la feuille vierge engluée située en dessous. Au centre de chaque piège est fixée une capsule attractive en caoutchouc, fournie par la station de phytophar- 


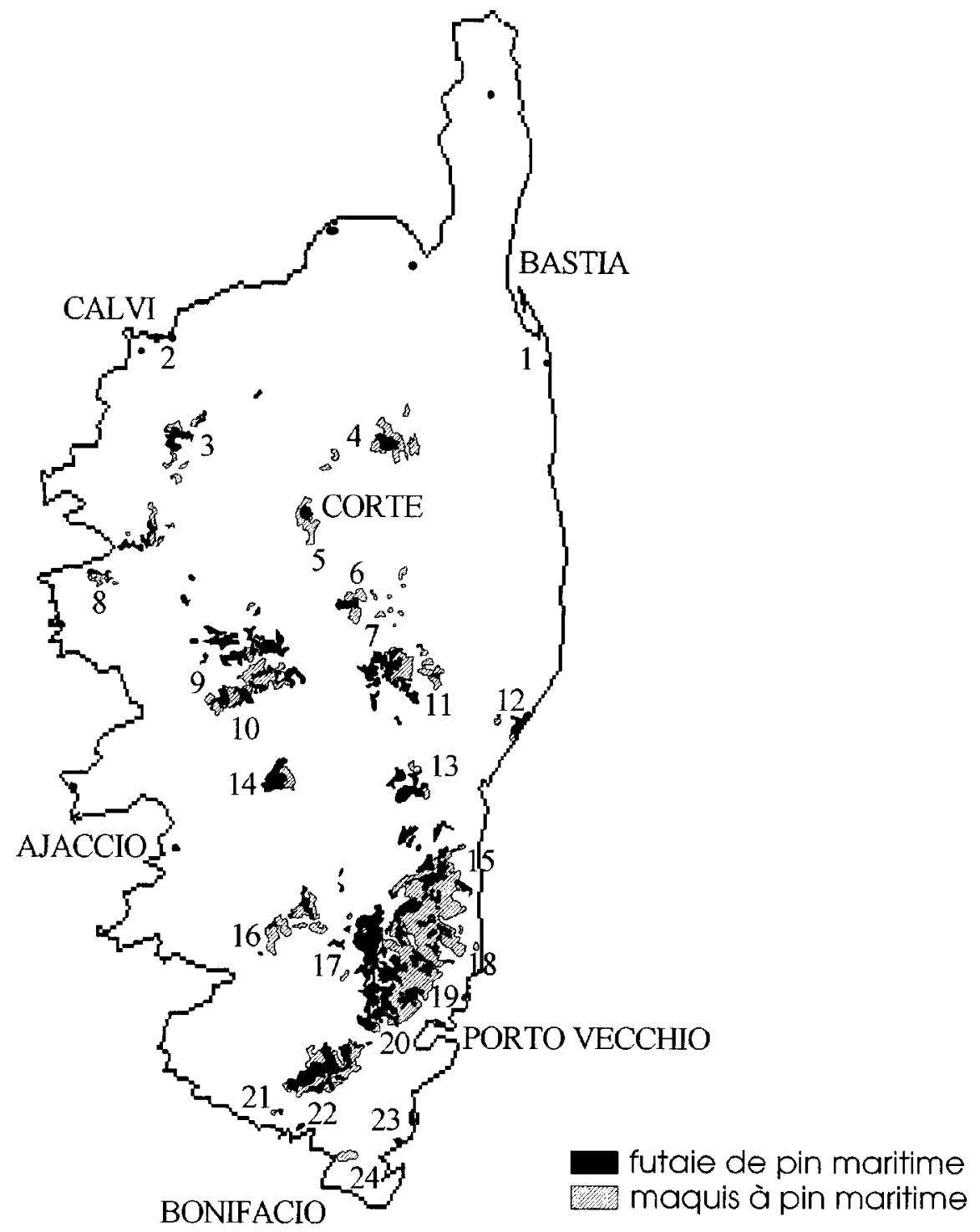

Fig 2. Répartition du pin maritime en Corse (d'après IFN, 1985) et situation des sites de piégeage.

macie (Inra, Versailles), chargée de $50 \mu \mathrm{g}$ de $(8 E, 10 E)$-3,7,9-trimethyl-8,10-dodecadien-6-one (Einhorn et al, 1990), dans sa configuration la plus active $3 S, 7 R$ (Jactel et al, 1994).

Dans chaque site, un seul piège a été installé, fixé sur le tronc d'un pin maritime à hauteur des yeux. La période de vol des mâles de la cochenille s'étale, aussi bien dans les Landes que dans les Maures et l'Esterel, du 25 février au 30 mars (Riom et Gerbinot, 1977) et la rémanence des diffuseurs de phéromone est de l'ordre de deux à trois semaines (Jactel et al, 1994). Tous les 
Tableau I. Caractéristiques des sites de piégeage sexuel et résultats des captures de Matsucoccus feytaudi.

\begin{tabular}{|c|c|c|c|c|c|}
\hline $\begin{array}{l}\text { Numéro } \\
\text { du piège }\end{array}$ & Commune & Forêt ou lieu-dit & $\begin{array}{l}\text { Altitude } \\
(m)\end{array}$ & $\begin{array}{c}\text { Type } \\
\text { forestier a }\end{array}$ & $\begin{array}{l}\text { Nombre de } \\
\text { mâles capturés }\end{array}$ \\
\hline & - & $-\quad--$ & - & - & - \\
\hline 1 & Borgo & Club de la Marana & 10 & FPM & 0 \\
\hline 2 & Calvi & Camping de la Pinède & 10 & FPM & 0 \\
\hline 3 & Calenzana & Forêt de Bonifatu & 700 & FPM & 0 \\
\hline 4 & Morosaglia & Forêt de Pineto & 300 & FPM & 311 \\
\hline 5 & Corte & Forêt de la Restonica & 600 & FPML & 0 \\
\hline 6 & Venaco & Venaco & 500 & FPML & 0 \\
\hline 7 & Venaco & Forêt de Cervello & 600 & FPM & 0 \\
\hline 8 & Piana & Forêt de Piana & 200 & FPM & 0 \\
\hline 9 & Azzana & Campu Vasalu & 300 & FPML & 0 \\
\hline 10 & Vero & Forêt de Vero & 600 & FPML & 0 \\
\hline 11 & Ghinosi & Domaine de Pinia & 10 & FPM & 0 \\
\hline 12 & Ghisoni & Domaine de Pinia & 10 & FPM & 0 \\
\hline 13 & Prunelli-di-Fiumorbo & San Gavino & 200 & FPM & 0 \\
\hline 14 & Bastelica & Forêt de Pineta & 700 & FPM & 0 \\
\hline 15 & Sari-Solenzara & Forêt de Tova & 100 & FPM & 0 \\
\hline 16 & Santa-Maria-Figaniella & Forêt de Valle Mala & 500 & MPM & 0 \\
\hline 17 & Levie & Forêt de Zonza & 800 & FPM & 0 \\
\hline 18 & Porto-Vecchio & Tagliu Rossu & 100 & MPM & 0 \\
\hline 19 & Porto-Vecchio & Sainte-Trinité & 40 & MPM & 0 \\
\hline 20 & Porto-Vecchio & Forêt de l'Ospedale & 350 & FPM & 0 \\
\hline 21 & Figari & Monaccia d'Aullene & 400 & FPM & 0 \\
\hline 22 & Figari & Cagna & 200 & FPM & 0 \\
\hline 23 & Bonifacio & Suartone & 100 & MPM & 0 \\
\hline 24 & Bonifacio & Ventilegne & 50 & MPM & 0 \\
\hline
\end{tabular}

a FPM : futaie de pin maritime ; FPML : futaie de pin maritime et pin laricio ; MPM : maquis de pin maritime.

pièges, relevés toutes les semaines, ont donc été mis en service au 2 mars 1994. Les diffuseurs de phéromone ont été changés une fois, au bout de 15 jours. Les derniers relevés ont été effectués du 30 mars au 5 avril. L'installation et les relevés des pièges ont été réalisés par les correspondants-observateurs du Département de la santé des forêts.

Toutes les feuilles transparentes ainsi relevées ont été recouvertes d'un film transparent et envoyées au laboratoire d'entomologie forestière (Inra, Bordeaux) pour identification. En cas de capture de mâles de $M$ feytaudi, des fragments d'écorce ont été prélevés sur l'arbre support du piège et sur les arbres voisins, puis expédiés au laboratoire d'entomologie forestière pour recherche des femelles et des formes larvaires fixées. Un échantillon des insectes récoltés a enfin été envoyé au laboratoire d'entomologie (muséum national d'Histoire naturelle, Paris) pour confirmer leur détermination.

\section{RÉSULTATS ET DISCUSSION}

Sur les 24 sites de piégeage retenus pour l'enquête, un seul, situé en Haute-Corse, dans la forêt de Pineto, a manifesté la présence de $M$ feytaudi (tableau I). Le piège correspondant $\left(n^{\circ} 4\right.$, fig 1 ) a capturé 311 
mâles de la cochenille, les deux tiers étant pris durant la première semaine de mars. Sur les échantillons d'écorce ont été trouvées une dizaine d'exuvies larvaires de deuxième stade et deux femelles.

II est donc désormais établi que $M$ feytaudi est présent en Corse. Ceci posé, la nature de la population isolée ainsi découverte reste inconnue ; d'elle dépend pourtant le risque de dépérissement qu'encourt le pin maritime dans cette région.

La première hypothèse concernant la nature de la population corse de $M$ feytaudi est qu'elle soit d'origine endémique, présente depuis de nombreuses années sur le site. Le faible niveau de capture, sans doute représentatif d'un faible niveau de population, est en effet du même ordre de grandeur que ceux observés pour une même dose de phéromone dans les Landes, région où $M$ feytaudi est endémique. Le grand morcellement de l'aire de répartition du pin maritime sur l'île, l'isolement géographique qu'impose le relief montagneux et la faible capacité de dissémination naturelle des cochenilles (Carle, 1973), essentiellement assurée par des larves transportées par le vent, pourraient alors expliquer que $M$ feytaudi n'ait été trouvé qu'en un seul site. D'après Baradat et Marpeau (1988), la race Corse de pin maritime correspondrait à l'hybridation de pins du sud-est de l'Espagne, apportés par les Phéniciens, avec des pins du nord-ouest de l'Italie, installés par les Génois. L'hétérogénéité génétique résultante pourrait alors expliquer la constitution de quelques peuplements isolés, présentant des arbres hôtes favorables au maintien de populations endémiques de cochenilles.

En tout état de cause, l'occurrence de dégâts notables imputables à ce type de population semble lointain. En effet, à l'échelle historique, aucune population endémique de $M$ feytaudi n'a manifesté d'évolution rapide vers un niveau épidémique.
La deuxième hypothèse relative à la nature de la population découverte en forêt de Pineto est qu'elle soit d'introduction récente. En effet, il serait étonnant que des populations endémiques, présentes depuis fort longtemps en Corse, n'aient pas fini par occuper la plupart des boisements de pin maritime de l'île, à l'exemple des populations marocaines qui occupent des forêts aussi isolées que celle de Tamjoute et du Rif au Maroc (Riom et Gerbinot, 1977). L'emplacement du site de Pineto, appartenant au seul massif de pins maritimes important du nord-est de la Corse, pourrait alors plaider en faveur d'une introduction accidentelle de cochenilles en provenance de Provence ou de Ligurie. Ce type de phénomène a déjà été invoqué pour interpréter le déclenchement de l'épidémie dans les Maures et l'Esterel (Schvester, 1971) et reste compatible avec le processus d'invasion actuel de la cochenille dans la partie orientale de l'aire du pin maritime.

D'une façon générale, les cochenilles se disséminent soit par leurs larves de premier stade, soit par l'intermédiaire de matériel végétal contaminé (Beardsley et Gonzalez, 1975). La dissémination des jeunes larves par le vent serait le plus souvent limitée, de l'ordre de quelques centaines de mètres comme pour Parlatoria pittospori (Mask), la cochenille du pin radiata (Timlin, 1964), c'est à dire beaucoup moins que les $150 \mathrm{~km}$ qui séparent la Corse du continent. Greathead (1972) fait bien mention d'une contamination de sites isolés, situés à plus de $200 \mathrm{~km}$ de foyers d'infestation, pour la cochenille de la canne à sucre Aucalapsis tegalensis (Zehntner). Dans ce dernier cas, les larves ont un comportement favorisant la dissémination anémophile, un tel transport bénéficiant en outre de conditions atmosphériques caractéristiques des zones tropicales. L'introduction de $M$ feytaudi correspondrait donc plus probablement à l'importation de matériel contaminé comme pour la plupart des cas d'introduction accidentelle de ravageurs 
forestiers (Speight et Wainhouse, 1989). La forêt de Pineto est d'ailleurs peu éloignée de Bastia, ville portuaire d'échanges avec l'Italie et le continent, mais également située au bord de l'axe routier reliant Bastia à Corte. Le caractère récent de cette introduction pourrait alors expliquer l'échec des précédentes enquêtes ainsi que l'absence de capture dans les autres sites de notre étude, sites non encore contaminés.

La majorité des problèmes économiques causés par les cochenilles interviennent lors de l'invasion de nouvelles zones géographiques (Beardsley et Gonzalez, 1975 ; Mendel et al, 1994). Issues des populations à l'état épidémique de Provence ou d'Italie, les cochenilles récemment introduites pourraient également manifester d'importantes capacités de reproduction. Enfin, les travaux portant sur la variabilité intraspécifique du pin maritime soulignent la grande sensibilité des provenances corses vis-àvis de $M$ feytaudi (Schvester et Ughetto, 1986, Harfouche et al, 1994). Dans cette situation, le risque de déclenchement d'une épidémie ne pourrait donc être rejeté, Carle (1973) estimant à deux ou trois ans le délai entre la première détection de la cochenille et l'apparition des premiers signes de dépérissement.

\section{CONCLUSION}

Pour plus de prudence, il paraît donc urgent de préciser l'ampleur et la nature effective des populations corses de $M$ feytaudi. Deux actions complémentaires semblent donc nécessaires. La première consisterait à préciser l'aire de répartition de la cochenille, voire à mettre en évidence d'autres foyers isolés, en installant un réseau de piégeage plus dense, centré sur la forêt de Pineto et le long de l'axe Bastia-Corte. La seconde, fondée sur l'analyse du génome de $M$ feytaudi, viserait à établir l'origine génétique des populations de l'île. Une étude du poly- morphisme génétique devrait en effet permettre d'évaluer les ressemblances existant entre les populations de cochenilles corses et les populations conspécifiques du reste de l'aire naturelle du pin maritime (Mendel et al, 1994). Proches des populations de Provence ou de Ligurie, les populations corses résulteraient probablement d'une introduction récente. Des différences importantes avec l'ensemble des populations de l'aire indiqueraient au contraire qu'elles sont de nature indigène.

\section{REMERCIEMENTS}

Cette étude n'aurait pu être menée à bien sans le concours de l'Échelon technique du Sud-Est du Département de la santé des forêts dirigé par $J$ Mirault, de ses correspondants-observateurs en Corse et tout particulièrement d'A Ceria. Qu'ils en soient remerciés. Nous sommes également très reconnaissants envers $\mathrm{J}$ Einhorn (station de phytopharmacie, Inra, Versailles) de nous avoir fourni la phéromone de synthèse, et I Foldi (laboratoire d'entomologie, muséum national d'Histoire naturelle, Paris) pour avoir effectué la détermination de nos échantillons de cochenilles.

\section{RÉFÉRENCES}

Baradat P, Marpeau-Bezard A (1988) Le pin maritime Pinus pinaster Ait : biologie et génétique des terpènes pour la connaissance et l'amélioration de l'espèce. Thèse, univ Bordeaux-l, $444 p$

Baradat $P$, Pastuska $P$ (1992) Le pin maritime. In : Amélioration des espèces végétales cultivées (A Gallais, H Bannerot, eds), Inra, Paris, 695-709

Baronio P, Butturini A (1988) Gli insetti nocivi al bosco Pinus spp. Monti e Boschi 6, I-VII|

Beardsley JW, Gonzalez RH (1975) The biology and ecology of armored scales. Ann Rev Entomol 20 , 47-73

Carle P (1968) Méthode d'obtention massive des pontes de Matsucoccus feytaudipar piégeage des femelles. Ann Sci For 25, 57-68

Carle P (1973) Le dépérissement du pin mésogéen en Provence. Rôle des insectes dans les modifications d'équilibre biologique des forêts envahies par Matsucoccus feytaudi Duc (Coccoidea: Margoridae). Thèse, univ Bordeaux-I, $174 \mathrm{p}$ 
Destremau DX (1974) Précisions sur les aires naturelles des principaux conifères marocains en vue de l'individualisation de provenances. Ann Rech For Maroc $14,5-90$

Einhorn J, Menassieu P, Malosse C, Ducrot PH (1990) Identification of the sex pheromone of the Maritime Pine Scale Matsucoccus feytaudi Duc. Tetrahedron Lett 46, 6633-6636

Greathead DJ (1972) Dispersal of the sugar-cane scale Aulacaspis tegalensis (Zhnt) by air currents. Bull Entomol Res 61, 547-558

Harfouche A, Baradat P, Kremer A (1995) Variabilité intraspécifique chez le pin maritime (Pinus pinaster Ait) dans le sud-est de la France. I. Variabilité des populations autochtones et de l'ensemble de l'aire de l'espèce. Ann Sci For 52, 307-328

IFN (1985) Carte des types de peuplements de la Corse. Inventaire forestier national, Paris

Jacte $H$, Menassieu P, Lettere M, Mori M, Einhorn J (1994) Field response of Maritime Pine Scale, Matsucoccus feytaudi Duc (Homoptera: Margarodidae), to synthetic sex pheromone stereoisomers. $J$ Chem Ecol 20, 2159-2170

Mendel Z, Nestel D, Gafny R (1994) Examination of the origin of the Israeli population of Matsucoccus josephi (Homoptera: Matsucoccidae) using random amplified polymorphic DNA-polymerase chain reaction method. Ann Entomol Soc Am 87, 165-169
Rieux R (1975) La spécificité alimentaire dans le genre Matsucoccus (Homoptera: Margarodidae) avec référence spéciale aux plantes hôtes de $M$ pini Green. Classement des Matsucoccus d'après leurs hôtes. Ann Sci For 32, 157-168

Riom J (1979) Étude biologique et écologique de la cochenille du pin maritime Matsucoccus feytaudi Ducasse, dans le sud-est de la France. Potentiel biotique et dynamique des populations. Ann Zool Ecol Anim 11, 397-456

Riom J, Gerbinot B (1977) Étude biologique et écologique de la cochenille du pin maritime Matsucoccus feytaudi Ducasse, dans le sud-est de la France. Biologie générale et phénologie. Ann Zool Ecol Anim 9, $11-50$

Schvester D (1971) Matsucoccus feytaudi Duc et «dépérissement" du pin maritime. Ann Zool Ecol Anim n ${ }^{\circ}$ hors-série, 139-150

Schvester D, Ughetto F (1986) Différences de sensibilité à Matsucoccus feytaudi Duc (Homoptera: Margarodidae) selon les provenances de pin maritime (Pinus pinaster Ait). Ann Sci For 43, 459-474

Speight MR, Wainhouse D (1989) Ecology and management of forest insects. Clarendon Press, Oxford, $374 \mathrm{p}$

Timlin JS (1964) The biology, bionomics and control of Parlatoria pittospori Mask: a pest on apples in New Zealand. N Z J Agr Res 7, 536-550 